\title{
Antimalarial Properties of Bredinin
}

\author{
PREDICTION BASED ON IDENTIFICATION OF DIFFERENCES
}

IN HUMAN HOST-PARASITE PURINE METABOLISM

\author{
H. K. Webster and J. M. Whaun, Department of Hematology, Walter Reed \\ Army Institute of Research, Washington, DC 20012
}

\begin{abstract}
A B S T R A C T Human malaria parasites (Plasmodium falciparum) grown in continuous erythrocyte culture utilize hypoxanthine for synthesis of both guanosine and adenosine nucleotides. Unlike the mature human erythrocyte, the malaria parasite depends on a constant supply of guanylates, primarily for synthesis of nucleic acids. This parasite specific requirement for guanylates led us to predict that a block in the hypoxanthine to quanosine monophosphate pathway would be selectively lethal to the parasite. Bredinin (4-carbamoyl-1- $\beta$-D-ribofuranyosyl-imidazolium - 5 olate) inhibited the synthesis of guanosine monophosphate from inosine monophosphate by parasitized erythrocytes. This block in guanylate synthesis was fatal to both a drug-sensitive (FCR-3) and a drug-resistant (VNS) strain of the malaria parasite at a bredinin concentration of $50 \mu \mathrm{M}$, arresting growth of the parasite at the trophozoite stage of development. These studies emphasize the essential role of guanylates and their synthesis from hypoxanthine in the metabolism of malaria parasite. They further suggest that bredinin or similar agents that selectively interfere with parasite guanylate metabolism may have potential for antimalarial chemotherapy.
\end{abstract}

\section{INTRODUCTION}

Truly rational approaches to antimalarial chemotherapy have been hampered by a lack of basic biochemical understanding of host-parasite relationships in human malaria infection. A major obstacle in the study of such host-parasite relationships was the lack of an effective in vitro culture system for human Plasmodia species. However, this problem was overcome with the

Dr. Webster's current address is U. S. Medical Component, Armed Forces Research Institute of Medical Sciences, (Bangkok, Thailand), APO San Francisco 96346.

Received for publication 11 August 1981 and in revised form 22 April 1982. development of in vitro culture techniques $(1,2)$ that permit continuous growth of the malaria parasite in human erythrocytes (RBC). ${ }^{1}$

We have used malaria culture techniques to study purine metabolism during the intraerythrocytic (IE) growth cycle of Plasmodium falciparum (malignant tertian malaria). We have concentrated on purine metabolism in our studies because of its importance to both the host RBC (3) and malaria parasite when studied in animal models (4-6). Identification of differences in host-parasite purine metabolism could present appropriate targets for design of new antimalarial chemotherapy.

Purine nucleotides are essential to both the RBC and parasite for energy metabolism, as enzyme cofactors and as intermediary metabolic regulators. However, only the parasite requires purine nucleotides for synthesis of nucleic acids (DNA and RNA), proteins, and folates $(7,8)$. We have paid particular attention to pathways for guanylate metabolism in malaria-infected RBC. A supply of guanine nucleotides appears to be a unique requirement of the parasite in contrast to the mature RBC where no established role for these compounds exists (9).

Using novel chromatographic techniques to study purine metabolism in malaria infected RBC in vitro, we have identified the major metabolic pathways used by IE $P$. falciparum to synthesize both guanosine and adenosine nucleotides from the precursor purine base, hypoxanthine (10). With the parasites' requirement for

\footnotetext{
${ }^{1}$ Abbreviations used in this paper: $\Sigma \mathrm{A}$, total adenylates; ECM, extracellular medium; $\Sigma \mathrm{G}$, total guanylates; GDP, guanosine diphosphate; GMP, guanosine monophosphate; GTP, guanosine triphosphate; Hct, hematocrit; HPLC, high performance liquid chromatography IE, intraerythrocytic; IMP, inosine monophosphate; PCA, perchloric acid; PRBC, parasitized RBC; RBC, erythrocytes; XMP, xanthosine monophosphate.
} 


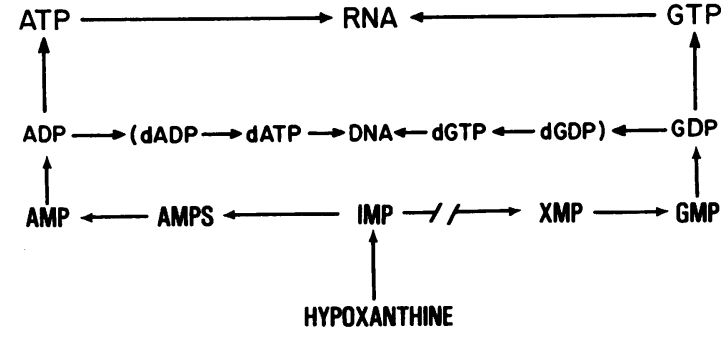

Figure 1 Pathways for synthesis of purine nucleotides in $P$. falciparum. Pathways for synthesis of adenosine and guanosine nucleotides from hypoxanthine via IMP are shown with the predicted site of action for bredinin involving the conversion enzyme, IMP dehydrogenase (EC 1.2.1.14). The relationships between purine ribonucleotides and synthesis of RNA and their conversion to deoxyribonucleotides for synthesis of DNA are also outlined. The malaria PRBC differs from the unparasitized host $R B C$ in the following ways: (a) presence of an adenylosuccinate (AMPS) pathway; (b) highly active guanylate pathway; $(c)$ utilization of hypoxanthine for synthesis of both adenosine and guanosine nucleotides; and $(d)$ active synthesis of both RNA and DNA.

guanylates and the absence of a known role for these compounds in mature RBC, we predicted that a block in the hypoxanthine-to-guanosine nucleotide pathway would be selectively lethal to the parasite. To test this hypothesis, bredinin, an imidazole nucleoside antibiotic, shown in leukemic and virus-infected cell lines to inhibit conversion of inosine monophosphate (IMP) to guanosine monophosphate (GMP) (11) (Fig. 1) was selected for use on $P$. falciparum-infected RBC in culture.

\section{METHODS}

Chemicals. Potassium phosphate (high performance liquid chromatography grade) was obtained from Fisher Scientific Co. (Pittsburgh, PA). Purine compounds of known purity were obtained from Sigma Chemical Co. (St. Louis, MO). Mini-Scint scintillation fluid was from Radiomatic Instruments \& Chemical Co., Inc. (Addison, IL) $\left[88^{-14} \mathrm{C}\right] \mathrm{Hy}-$ poxanthine $(52.8 \mathrm{mCi} / \mathrm{mmol})$ was obtained from New England Nuclear (Boston, MA). [G- $\left.{ }^{3} \mathrm{H}\right]$ Hypoxanthine (1 Ci/ $\mathrm{mmol}$ ) and $\mathrm{L}-\left[4,5-{ }^{3} \mathrm{H}\right]$ isoleucine $(15-40 \mathrm{Ci} / \mathrm{mmol})$ were purchased from Amersham Corp. (Arlington Heights, IL). RPMI 1640 media were prepared by Gibco Laboratories, Grand Island Biological Co. (Grand Island, NY). Bredinin (4-carbamoyl-1- $\beta$-D-ribofuranosyl-imidazolium-5-olate) was purchased from Calbiochem-Behring Corp., American Hoechst Corp. (San Diego, CA).

Malaria culture. Two strains of $P$. falciparum were used in these studies: the FCR-3 strain, which is chloroquine sensitive, obtained from David Haynes (Walter Reed Army Institute of Research); and the Vietnam Smith (VNS) strain, which is chloroquine resistant, was provided by George Childs (Walter Reed Army Institute of Research). These strains have been described in the literature $(12,13)$ and used in human volunteer studies (14).

Experimental assays were done as follows. Culture flasks $\left(25 \mathrm{~cm}^{2}\right)$ containing $5 \mathrm{ml}$ of a $6 \% \mathrm{RBC}(\mathrm{O}+$ donor) suspension in RPMI 1640 medium with $10 \%$ serum (A+ donor) were seeded with infected RBC from a maintenance culture so as to give an initial parasitemia of $0.5 \%$. Each flask was purged with a gas mixture $\left(90 \% \mathrm{~N}_{2}, 5 \% \mathrm{O}_{2}, 5 \% \mathrm{CO}_{2}\right)$ and tightly capped. Cultures were incubated at $37^{\circ} \mathrm{C}$ in an atmosphere of the same gas mixture. These cultures were observed to be of asynchronous growth and to yield a 10-fold increase in parasitemia over a 48-h period. The parasite undergoes a complete IE growth cycle (schizogony) in $\sim 42 \mathrm{~h}$. After 24-h incubation, a portion (3-4 ml) of the culture extracellular medium (ECM) was removed and replaced with an equal volume of fresh medium. To adjust for variation in parasite growth in individual flasks during the first $24 \mathrm{~h}$ of incubation the contents of all parasitized RBC (PRBC) flasks for use in a given experiment were mixed in a large sterile flask and redistributed in aliquots to individual flasks. After the medium change and redistribution of cultures the desired isotope and inhibitor were added. Incubation of the cultures was then continued for various times. Control cultures consisted of PRBC without the inhibitor and unparasitized RBC with and without the inhibitor. Control cultures were processed at the same time and in the same manner as the test cultures. Cultures were set up in duplicate or triplicate within a given experiment. This experimental approach was referred to as a unit flask assay. Each group of similarly treated cultures was incubated under conditions suitable for a 10-fold increase in parasitemia during which a complete IE malaria growth cycle occurred $(48 \mathrm{~h})$. In other experiments, the contents of several (3-4) unit flasks were pooled before extraction (pooled flask assay). In this approach, additions of isotope and inhibitor were made to the individual flasks following a second medium change $(48 \mathrm{~h})$ and incubation was continued for $3 \mathrm{~h}$.

Preparation of cell extracts. Malaria cultures were removed from incubation and processed as follows. The contents of each unit flask $(5 \mathrm{ml})$ were pipetted into precooled plastic tubes $(75 \times 100 \mathrm{~mm})$ and centrifuged $(270 \mathrm{~g})$ for 5 min $\left(4^{\circ} \mathrm{C}\right)$, to separate the RBC from their ECM. $3.9 \mathrm{ml}$ of medium was removed and $2 \mathrm{ml}$ of this was pipetted into 1 $\mathrm{ml}$ of $1 \mathrm{M}$ perchloric acid (PCA), mixed vigorously, and the extraction mixture placed on ice. The additional $1.9 \mathrm{ml} \mathrm{ECM}$ was saved for other experiments. The RBC button was resuspended in the remaining $1.1 \mathrm{ml}$ of ECM. $1 \mathrm{ml}$ of the RBC suspension was pipetted into $1 \mathrm{ml} 1 \mathrm{M}$ PCA, mixed, and placed on ice. Extraction was continued for $20 \mathrm{~min}$ after which the tubes were centrifuged $\left(1,200 \mathrm{~g}, 10 \mathrm{~min}, 4^{\circ} \mathrm{C}\right)$ to remove the acid precipitated material. The clear supernatant was removed volumetrically and neutralized with $10 \mathrm{M}$ KOH. The resulting potassium perchlorate salt was removed by centrifugation $\left(12,000 \mathrm{~g}, 10 \mathrm{~min}, 4^{\circ} \mathrm{C}\right)$, the supernatant recovered, frozen and stored at $-70^{\circ} \mathrm{C}$ until analysis. $\mathrm{He}$ matocrits (Hct) and smears for Giemsa staining were prepared from the $0.1 \mathrm{ml}$ of remaining suspended cells. Pooled flasks were processed in a similar manner except that the starting culture volume was $15-20 \mathrm{ml}$.

Ribonucleosides and bases were found predominantly in the ECM, whereas ribonucleotides were almost all found intracellularly. Extraction with PCA was found to give good recoveries ( $294 \%$, using radiolabeled standards) of all ribonucleotides reported. Deoxyribonucleotides, for which PCA extraction is not particularly efficient, were not reported.

Determination of radioactivity incorporated into nucleic acids was done by alkali solubilization of the acid-insoluble material formed during PCA-extraction of PRBC. An aliquot of solubilized pellet was counted by liquid scintillation counting. Protosol (New England Nuclear) was used to dis- 
solve the twice-acid-washed cell pellet. This technique measures total nucleic acids and, therefore, provides an estimate of label incorporated into both DNA and RNA.

Quantitation of purine compounds by simultaneous UVradioactivity HPLC. Purines were assayed using an HPLC system that simultaneously measured the concentration and radioactivity of separated components (15). Basically, the HPLC system consisted of the following: a model 3500B HPLC (Spectra-Physics Inc., Mountain View, CA) fitted with a solvent programmer and sample injector value; model 152 dual wavelength (254 and $280 \mathrm{~nm}$ ) detector with $20 \mu \mathrm{l}$ analytical flow cell (Altex Company, Tarrington, CT) connected to a System I Computing Integrator (Spectra-Physics) and strip-chart recorder (Hewlett-Packard Co., Palo Alto, CA); a FLO-ONE radioactivity flow detector with a 500$\mu l$ flow cell (Radiomatic Instruments \& Chemical Co.). The radioactivity flow detector was connected in series with the UV-detector (column effluent passed first through the UV flow cell, then through the radioactivity flow cell). The radioactivity detector unit mixed the column effluent with scintillation fluid (predetermined ratio) for counting in the flow cell.

Purine nucleosides and bases were separated by reversedphase HPLC using an Ultrasphere-5 ODS column (Altex Company). Purine nucleotides were separated by anion-exchange HPLC with a Partisil-10 SAX microparticulate column (Whatman, Inc., Chemical Separation Div., Clifton, $\mathrm{NJ}$ ).

The neutralized PCA extracts were thawed and injected directly onto the HPLC column using a valve injector (Valco

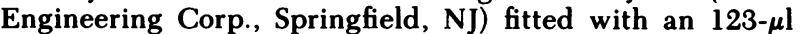
loop. Samples prepared in this manner contained sufficient amounts of ribonucleotides for detection and accurate quantitation. Deoxyribonucleotides were separated from their corresponding ribonucleotides by both the reversed-phase and anion-exchange HPLC techniques. Deoxyribonucleotide forms were found to elute distinctly just after the corresponding ribonucleotide form. These separation profiles were confirmed using commercial (Sigma Chemical Co.) purine standards. Deoxyribonucleotides were not, however, detected routinely in sample extracts, due to their low intrinsic pool levels and the noted questionable recovery with PCA extraction.

Concentrations were determined by an external standard method based on absorbance (254 $\mathrm{nm}$ ) using solutions of purine standards of known purity. For radioactivity measurements, peak areas were machine-integrated and recorded as total counts per radioactivity peak.

Microtiter assay. Evaluation of bredinin's antimalarial activity in terms of dose response was done by microtitration techniques essentially as described by Desjardins and colleagues (14). Microtiter plates (96 wells arranged in an 8 $\times 12$ matrix) were supported in specially designed humidified air tight boxes, flushed with gas mixture $\left(90 \% \mathrm{~N}_{2}, 5 \%\right.$ $\mathrm{O}_{2}, 5 \% \mathrm{CO}_{2}$ ) and incubated at $37^{\circ} \mathrm{C}$. Each microtiter well contained $200 \mu$ l total volume: $25 \mu$ l of RPMI 1640 medium ( \pm varying dilutions of drug); and, a $175 \mu$ l volume of a $1 \%$ PRBC suspension (initial parasitemia of $0.5 \%$ PRBC). Separate microtiter plates containing equivalent volumes of unparasitized RBC suspension were set up as RBC controls. After $24 \mathrm{~h}$ incubation at $37^{\circ} \mathrm{C}, 25 \mu \mathrm{l}$ of $\left[{ }^{3} \mathrm{H}\right]$ hypoxanthine $\left(0.5 \mu \mathrm{Ci} /\right.$ well) or $25 \mu \mathrm{l}$ of $\left[{ }^{3} \mathrm{H}\right]$ isoleucine $(1.0 \mu \mathrm{Ci} /$ well $) \mathrm{di}$ luted in RPMI medium was added to assess incorporation of label into nucleic acid or protein, respectively. Microtiter plates were then incubated at $37^{\circ} \mathrm{C}$ for an additional $24 \mathrm{~h}$ and harvested on a MASH II automated cell harvester (Microbiological Associate, Bethesda, MD) for liquid scintilla- tion counting. Test and control microtiter plates were set up in triplicates. Parasite growth was assessed by microscopic evaluation of Giemsa-stained thin-smears.

\section{RESULTS}

Effect of bredinin on purine nucleotide synthesis. Fig. $2(a-c)$, illustrates the effect of bredinin on the synthesis of purine ribonucleotides from [8${ }^{14} \mathrm{Cl}$ hypoxanthine as identified by simultaneous UVradioactivity HPLC. Unparasitized RBC (control) incorporated $\left[{ }^{14} \mathrm{C}\right]$ hy poxanthine primarily into IMP (Fig. 2a). It should be noted that the donor RBC used for these cultures had been stored (acid citrate dextrose donor units) for 8-12 d before use and detectable levels of IMP had accumulated.

Although some synthesis of guanosine nucleotides from IMP occurred in uninfected RBC under the culture conditions used (see peaks 5 guanosine diphosphate (GDP) and 7 guanosine triphosphate (GTP), Fig. 2a), none of the label from hypoxanthine was incorporated into adenosine nucleotides. PRBC actively incorporated $\left[{ }^{14} \mathrm{C}\right]$ hypoxanthine into both guanosine and adenosine nucleotides via IMP (Fig. 2b). Adenylate synthesis from IMP, which occurred only in malariainfected RBC, also accounted for the appearance of radioactivity in pyridine nucleotides (radioactivity peak 1 was NAD, Fig. 1b). In the presence of bredinin $(50 \mu \mathrm{M})$ there was decreased incorporation of $\left[{ }^{14} \mathrm{C}\right]$ hypoxanthine into the guanosine nucleotides of PRBC (Fig. lc; compare radioactivity peaks 5 and 7 , Figs. $1 \mathrm{~b}$ and $1 \mathrm{c})$.

Table I shows the quantitative results obtained using individual cultures (triplicates) to which both the isotope and drug were added following the medium change and redistribution of the PRBC volume (unit flask assay). The parasitemia (percent infected RBC and distribution of IE parasite growth stages) for each culture was equivalent at the start of the experiment. The data were obtained by integration of chromatographic peaks of the HPLC eluation profiles. In the presence of bredinin $(50 \mu \mathrm{M})$ there was a decrease in the incorporation $(85 \%, P<0.001)$ of $\left[{ }^{14} \mathrm{C}\right]$ hypoxanthine into guanylates (GDP, GTP, $\left.\Sigma \mathrm{G}\right)$ of PRBC, compared with untreated PRBC, after 24-h incubation in the presence of labeled hypoxanthine. There was also a decrease in GDP $(25 \%, P<0.01)$ and GTP $(39 \%, P<0.001)$ pool levels in the infected RBC cultures. Production of IMP from hypoxanthine was not affected by bredinin, indicating a block in the synthesis of GMP from IMP. Hypoxanthine levels were found to be higher in extracts of ECM from bredinintreated PRBC compared with untreated PRBC $(11 \pm 1$ vs. $7 \pm 1 \mathrm{nmol} / \mathrm{ml}, P<0.02$; and $4,087 \pm 38$ vs. $2,910 \pm 138$ as regards radioactivity per chromatographic peak). 

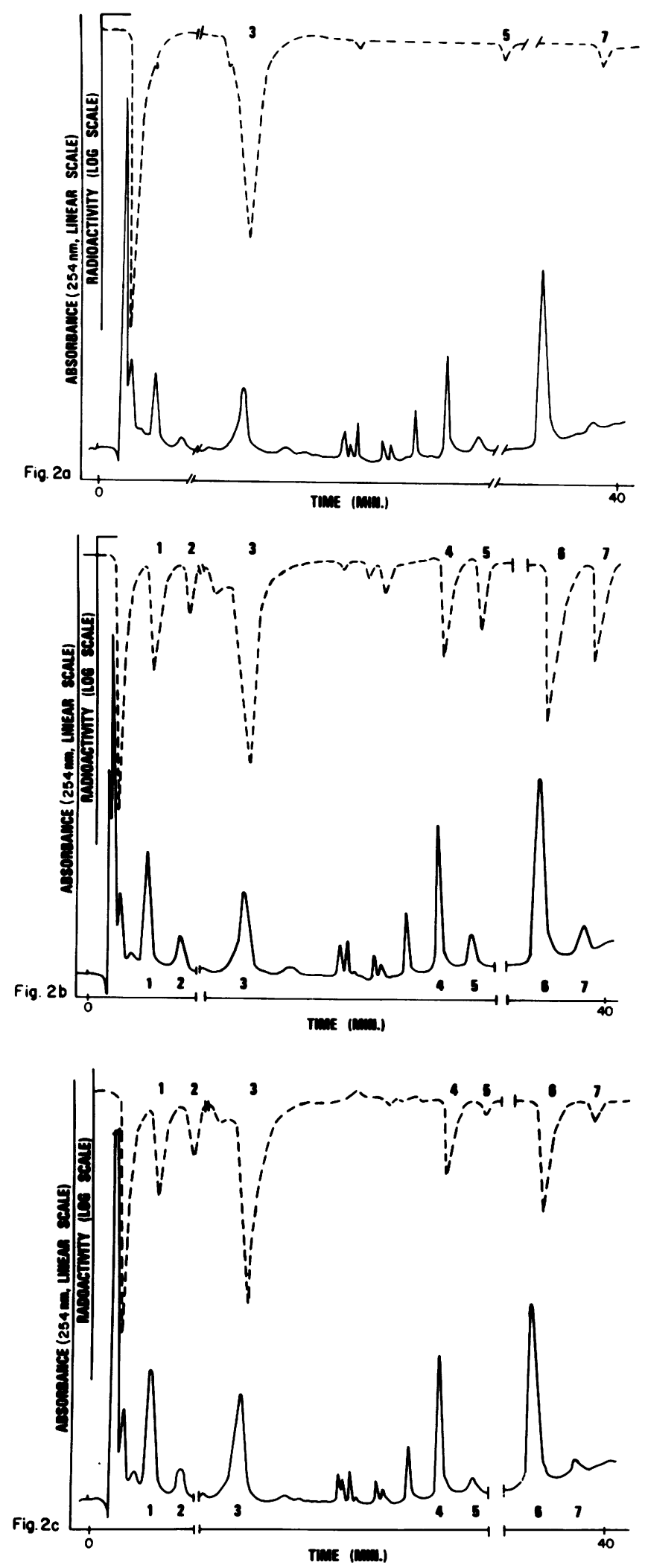
TABLE I

Effect of Bredinin (50 $\mu \mathrm{M})$ on Incorporation of $\left[{ }^{14} \mathrm{C}\right]$ Hypoxanthine into Purine Ribonucleotides and on Levels of Ribonucleotides in PRBC

\begin{tabular}{|c|c|c|c|c|c|c|c|c|c|c|}
\hline Culture conditions & & ATP & ADP & AMP & GTP & GDP & IMP & $\Sigma \Lambda$ & $\Sigma G$ & $(\Sigma \mathbf{A} / \Sigma \mathbf{G})$ \\
\hline \multirow{3}{*}{$\begin{array}{l}\text { Malaria-infected, } \\
\text { untreated RBC } \\
(50 \mathrm{~h})\end{array}$} & Nanomoles & $\begin{array}{c}1,226 ! \\
\pm 92\end{array}$ & $\begin{array}{r}126 \\
\pm 9\end{array}$ & $\begin{array}{r}10 \\
\pm 1\end{array}$ & $\begin{array}{l}87 \S \\
\pm 2\end{array}$ & $\begin{array}{l}12^{11} \\
\pm 1\end{array}$ & $\begin{array}{r}369 \\
\pm 15\end{array}$ & $\begin{array}{r}1,365 \\
\pm 87\end{array}$ & $\begin{array}{l}99 \S \\
\pm 2\end{array}$ & 14 \\
\hline & Radioactivity $\pi$ & $\begin{array}{l}1,666 \\
\pm 116\end{array}$ & $\begin{array}{r}159 \\
\pm 18\end{array}$ & $\begin{array}{r}135 \\
\pm 32\end{array}$ & $\begin{array}{l}590 \S \\
\pm 12\end{array}$ & $\begin{array}{l}113^{11} \\
\pm 15\end{array}$ & $\begin{array}{r}10,685 \\
\pm 1,048\end{array}$ & $\begin{array}{l}2,010 \\
\pm 107\end{array}$ & $\begin{array}{l}7038 \\
\pm 13\end{array}$ & 3 \\
\hline & Specific activity ${ }^{\bullet}$ & 1 & 1 & 13 & 7 & 9 & 29 & & & \\
\hline \multirow{3}{*}{$\begin{array}{l}\text { Malaria-infected } \\
\text { bredinin-treated } \\
\text { RBC }(50 \mathrm{~h})\end{array}$} & Nanomoles & $\begin{array}{l}1,249 \\
\pm 124\end{array}$ & $\begin{array}{r}115 \\
\pm 2\end{array}$ & $\begin{array}{r}13 \\
\pm 3\end{array}$ & $\begin{array}{l}53 \S \\
\pm 2\end{array}$ & $\begin{array}{r}9^{\prime \prime} \\
\pm 1\end{array}$ & $\begin{array}{r}361 \\
\pm 54\end{array}$ & $\begin{array}{l}1,376 \\
\pm 123\end{array}$ & $\begin{array}{l}62 \S \\
\pm 2\end{array}$ & 22 \\
\hline & Radioactivity & $\begin{array}{l}1,397 \\
\pm 125\end{array}$ & $\begin{array}{r}156 \\
\pm 9\end{array}$ & $\begin{array}{r}185 \\
\pm 35\end{array}$ & $\begin{aligned} & 70 \S \\
\pm & 22\end{aligned}$ & $\begin{array}{l}39^{\prime \prime} \\
\pm 11\end{array}$ & $\begin{array}{r}10,065 \\
\pm 1,502\end{array}$ & $\begin{array}{l}1,688 \\
\pm 106\end{array}$ & $\begin{array}{l}109 \S \\
\pm 33\end{array}$ & 16 \\
\hline & Specific activity & 1 & 1 & 14 & 1 & 4 & 28 & & & \\
\hline
\end{tabular}

\footnotetext{
- Nanomoles per milliliter RBC (nucleotides based on Hct). with differing parasitemias.

\& Indicate significant $(P<0.001)$ differences between control and treated values using the Student's $t$ test.

"Indicate signficant $(P<0.01)$ differences between control and treated values using the Student's $t$ test.

I Integrated radioactive counts (area) per chromatography peak.

- Specific activity, radioactivity per nanomole.
}

IValues are means $( \pm \mathrm{SEM})$ (triplicate cultures with similar parasitemias) and are representative of results observed in seven experiments

Inosine was not detected in ECM from cultures of PRBC.

The exact site of inhibition in the guanylate pathway $(\mathrm{Hyp} \rightarrow \mathrm{IMP} \rightarrow \mathrm{XMP} \rightarrow \mathrm{GMP} \rightarrow \mathrm{GDP} \rightarrow \mathrm{GTP})$ was not assessed in these experiments since detectable levels of GMP or xanthosine monophosphate (XMP) were not obtained under the experimental conditions of the unit flask assay. This problem was resolved with the pooled flask assay reported below. An inhibitory effect by bredinin on guanylate metabolism in unparasitized RBC controls was also observed although the significance of reduced guanylates for RBC function if any, was not apparent. Control RBC incorporated quantitatively less radioactivity in guanylates than PRBC even though the Hct in each were equivalent ( $\Sigma G$ for
PRBC was sevenfold greater than $\Sigma G$ for RBC, with a Het of 12; RBC data not shown).

Bredinin did not appear to affect directly adenylate metabolism in PRBC (Table I). The reduced amount of radioactivity in adenylates $(\Sigma \mathrm{A})$ noted in the bredinin-treated PRBC was thought to reflect primarily a decreased availability of GTP required in the synthesis of adenylosuccinate from IMP. The increased levels of AMP in treated PRBC was most likely due to increased catabolism of ATP.

That the block in guanylate synthesis produced by bredinin affected parasite's nucleic acid synthesis was evident from comparison of incorporated radioactivity into the acid-insoluble residue (pellets) following PCA extraction. In this series of experiments bredinin (50

Figure 2 (a-c) Purine nucleotide profiles obtained by simultaneous UV-radioactivity HPLC. Neutralized PCA extracts prepared from malaria/RBC cultures and injected onto an anionexchange column for separation of purine nucleotides by the HPLC procedure described in the text. This procedure allows the simultaneous quantitation of both concentration $(254 \mathrm{~nm})$ and radioactivity of separated purine components. Absorbance is traced on a linear scale, whereas the radioactivity trace is on a logarithmic scale. Peaks were computer integrated. $(a)$ Profile of uninfected RBC control culture incubated with $\left[{ }^{14} \mathrm{C}\right]$ hypoxanthine for $24 \mathrm{~h}$. (b) Profile of PRBC (4.2\%) after 24-h incubation with $\left[{ }^{14} \mathrm{C}\right]$ hypoxanthine. (c) Profile of bredinin-treated PRBC after $24-h$ incubation with $\left[{ }^{14} \mathrm{C}\right]$ hypoxanthine plus bredinin $(50 \mu \mathrm{M})$. Peaks were identified by correspondence to standards. Peak numbers: (1) NAD; (2) AMP; (3) IMP; (4) ADP; (5) GDP; (6) ATP; and (7) GTP. 
$\mu \mathrm{M})$ and $\left[{ }^{14} \mathrm{C}\right]$ hypoxanthine were added together to infected cultures ( $2.2 \%$ PRBC) during a medium change. The amount of radioactivity remaining in the acid-insoluble residue was used as a measure of nucleic acid synthesis. Radioactivity observed in residue aliquots from PRBC, bredinin-treated PRBC and unpar-

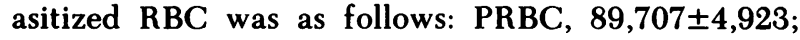
bredinin-treated PRBC, 64,761 $\pm 2,303$; RBC, 341 \pm 32 (dpm \pm SEM, $n=3 P<0.05)$. This reflects a $28 \%$ decrease of incorporated label into nucleic acids by bredinin-treated PRBC relative to untreated PRBC during a 24-h incubation period.

The experiments reported thus far all involved the FCR-3 strain of $P$. falciparum that was chloroquine sensitive. It was of interest to examine the effect of bredinin on a chloroquine-resistant malaria parasite, the VNS strain. A unit flask assay, similar to that reported above with the FCR-3 strain, was done using the VNS strain. Cultures for both bredinin-treated (50 $\mu \mathrm{M})$ and untreated PRBC were set up in quadruplicate with extracts of individual cultures analyzed by HPLC as described under Methods. A decrease in both concentration and incorporated radioactivity of guanylates was observed $(\Sigma \mathrm{G}, 52 \pm 6 \mathrm{nmol} / \mathrm{ml} \mathrm{RBC}$ vs. $88 \pm 9$ $\mathrm{nmol} / \mathrm{ml} \mathrm{RBC}, 41 \%$ decrease, $P<0.005 ; \Sigma \mathrm{G} 128 \pm 37$ radioactivity/peak vs. $800 \pm 88$ radioactivity/peak, $84 \%$ decrease, $P<0.005$ ); for bredinin-treated PRBC compared with untreated PRBC. There were no major observed differences in the overall purine metabolism of labeled $\left[{ }^{14} \mathrm{C}\right]$ hypoxanthine noted between the two strains of $P$. falciparum.
Although the results from the unit flasks assay approach demonstrate that bredinin interferes with the synthesis of GMP from IMP by malaria PRBC, it does not establish which enzyme in this pathway, IMP dehydrogenase or GMP synthetase, was inhibited. As noted above for conditions of the unit flasks assay, levels of XMP and GMP were not detected. Presumably, this was due to the relatively small numbers of erythrocytes $(H c t \simeq 12)$ and a sufficiently rapid turnover of these nucleotide monophosphates to preclude their accumulation. Nonetheless, if bredinin did interfere with GMP synthetase, some accumulation of XMP would be anticipated, particularly in view of the absence of a xanthine oxidase in human RBC and the fact that no build-up of labeled uric acid during the culture period was observed in chromatograms of ECM from bredinin-treated PRBC.

As a means of evaluating the levels of GMP and XMP during parasite growth, a pooled flask assay was used. In this way it was possible to obtain Hct $\simeq 30$. Note that in this assay the inhibitor and isotope were added after the second medium change (higher starting parasitemia) and then incubation continued for 3 h. The parasitemias were equivalent in both treated and untreated cultures, due to the pooling and redistribution done at the medium change. There was no significant change in the parasitemia of any cultures during the 3 -h incubation $(5.8 \pm 0.8 \%$ PRBC; $n=3)$. Table II shows that the amount of radioactivity from $\left[{ }^{14} \mathrm{C}\right]$ hypoxanthine incorporated into both XMP and GMP was sharply decreased in bredinin-treated PRBC

TABLE II

Effect of Bredinin $(50 \mu \mathrm{M})$ on Incorporation of $\left[{ }^{14} \mathrm{C}\right]$ Hypoxanthine into Purine Ribonucleotides of the Guanylate Pathway

\begin{tabular}{|c|c|c|c|c|c|c|}
\hline & & IMP & XMP & GMP & GDP & GTP \\
\hline \multirow{5}{*}{$\begin{array}{l}\text { Malaria-infected } \\
\text { untreated RBC } \\
(3 \mathrm{~h})\end{array}$} & Nanomoles ${ }^{\circ}$ & $217 \ddagger$ & 4 & 4 & 20 & 90 \\
\hline & & \pm 3 & \pm 0.3 & \pm 0.1 & \pm 0.3 & \pm 1 \\
\hline & Radioactivity $\S$ & 56,865 & 196 & 46 & 264 & 1,632 \\
\hline & & \pm 119 & \pm 5 & \pm 5 & \pm 15 & \pm 79 \\
\hline & Specific activity" & 262 & 43 & 11 & 13 & 18 \\
\hline \multirow{6}{*}{$\begin{array}{l}\text { Malaria-infected } \\
\text { bredinin-treated } \\
\text { RBC }(3 \mathrm{~h})\end{array}$} & Nanomoles & 186 & 3 & 3 & 19 & 71 \\
\hline & & \pm 5 & \pm 0.2 & \pm 0.1 & \pm 0.2 & \pm 1 \\
\hline & Radioactivity & 53,850 & 16 & 19 & 68 & 103 \\
\hline & & \pm 159 & \pm 2 & \pm 6 & \pm 3 & \pm 5 \\
\hline & & & $(P<0.001) \pi$ & $(P<0.02)$ & $(P<0.001)$ & $(P<0.001)$ \\
\hline & Specific activity & 290 & 5 & 6 & 4 & 2 \\
\hline
\end{tabular}

- Nanomoles per milliliter RBC (nucleotides based on Hct).

$\ddagger$ Values are means of three determinations using pooled flask assay.

$\$$ Integrated ratioactive counts (area) per chromatography peak.

"Specific activity, radioactivity per nanomole.

I Indicate significant differences between control and treated values using the Student's $t$ test. 
as compared with untreated PRBC. This effect of bredinin was particularly evident when the specific activities for GMP and XMP were compared for treated vs. untreated PRBC (Table II). Although the differences in actual concentration (nanomoles per milliliter RBC of GMP and XMP) were small, the sharply decreased specific activities for these mononucleotides in bredinin-treated PRBC indicates a considerable reduction in the synthesis of new XMP and GMP during the $3-h$ incubation period. These data suggest, therefore, that bredinin acts on IMP dehydrogenase.

Rescue of bredinin-treated PRBC by exogenous GMP. Table III shows that addition of GMP $(50 \mu \mathrm{M})$ to bredinin-treated $(50 \mu \mathrm{M})$ PRBC reverses inhibition as measured both by incorporation of $\left[{ }^{3} \mathrm{H}\right]$ isoleucine into protein and parasite growth. There was significant $(P<0.001)$ incorporation of isoleucine into PRBC accompanied by parasite growth when bredinin-treated PRBC were incubated in the presence of equimolar GMP. Lower concentrations of GMP were less effective. Reversal of the inhibition of GMP synthesis from hypoxanthine in the presence of exogenously added GMP was not noted (observation based on unit flask assay). The GMP provides an alternative source of purine base for synthesis of guanylates by the parasite (viz: G $\rightarrow$ GMP $\rightarrow$ GDP $\rightarrow$ GTP). There were no detectable levels of guanine or guanosine in the ECM under the conditions of culture used for these studies. These data indicate both that hypoxanthine is the source of parasite GMP and that guanylates are essential to parasite growth.

Use of the microtiter assay to confirm the antimalarial activity of bredinin. To assess the effects of bredinin on malaria parasite survival and growth in vitro, a microtiter assay technique was used. Uptake of $\left[{ }^{3} \mathrm{H}\right]$ hy poxanthine into precipitable material (nucleic acids) served as a biochemical indicator of parasite growth and microscopic examination of Giemsa-stained smears prepared from each microtiter well permitted evaluation of parasite maturation and proliferation. Fig. 3 shows the effect of varying concentrations of

TABLE III

Rescue of Bredinin-treated PRBC by Exogenous GMP ( $n=3)$

\begin{tabular}{|c|c|c|}
\hline & $\begin{array}{l}\text { PHJlsoleucine } \\
\text { uptake }\end{array}$ & PRBC \\
\hline & $d p m \pm S E M$ & $\%$ counts $\pm S E M$ \\
\hline $\begin{array}{l}\text { Control } \\
\text { Bredinin-treated }\end{array}$ & $7,333 \pm 423$ & $4.2 \pm 0.4$ \\
\hline $\begin{array}{l}(50 \mu \mathrm{M}) \\
\text { Bredinin-treated + GMP }\end{array}$ & $2,232 \pm 621^{\circ}$ & $0.43 \pm 0.1$ \\
\hline$(50 \mu \mathrm{M})$ & $7,397 \pm 127^{\circ}$ & $3.5 \pm 0.2$ \\
\hline
\end{tabular}

- $P<0.001$. bredinin on parasite growth and metabolism. There was a precipitous decrease in $\left[{ }^{3} \mathrm{H}\right]$ hypoxanthine uptake and in the percentage of PRBC at bredinin concentrations of $50 \mu \mathrm{M}$ and higher, although a decrease in $\left[{ }^{3} \mathrm{H}\right]$ hypoxanthine uptake was initially noted at a bredinin concentration of $5 \mu \mathrm{M}$.

Microscopic examination of Giemsa-stained smears showed a graded effect on parasite morphology that correlated with the concentration of bredinin. Although some change in nuclear and cytoplasmic organization was observed at $0.5 \mu \mathrm{M}$ bredinin, major effects became apparent at concentrations of $50 \mu \mathrm{M}$ and higher. The most notable feature at low concentrations $(5 \mu \mathrm{M}$ and $0.5 \mu \mathrm{M})$ was an increase in the number of schizonts based on the differential count. (A parasite differential count is made during microscopic examination of the Giemsa smear.) The number of PRBC in $100 \mathrm{RBC}$ gives the percent PRBC. The $P R B C$ are counted as containing one of three parasite growth stages that characterize IE schizogony: young trophozoites ("ring"), mature trophozoites, or schizonts. At bredinin concentrations $\geq 50 \mu \mathrm{M}$, however, the schizont forms of the parasite were observed to be absent or greatly reduced in all microscopic preparations. The predominant growth stage at these concentrations $(>50 \mu \mathrm{M})$ was the mature trophozoite. This suggests that the parasite's growth is arrested during the middle to late trophozoite stage of development. The trophozoites were characterized by the appearance of pyknotic nuclei, a decrease in parasite cytoplasm and cytoplasmic vacuolation, and a decrease in overall size of the parasite body relative to the host RBC.

\section{DISCUSSION}

Our results clearly demonstrate that bredinin is an active antimalarial agent in vitro against both a drug (chloroquine, quinine, and pyrimethamine)-susceptible strain and drug-resistant strain of $P$. falciparum. The primary biochemical site of bredinin's action appears to involve a block in the synthesis of GMP from IMP due to inhibition IMP dehydrogenase. This decrease in the supply of guanosine nucleotides to the rapidly proliferating malaria parasite resulted in impaired nucleic acid synthesis with an arrest of parasitic growth at the trophozoite stage. Although interference with nucleic acid synthesis by bredinin was assumed to be the major biochemical lesion to the parasite, there are several other metabolic effects that can be attributed to decreased availability of guanylates. Guanosine nucleotides are important cofactors and substrates for a number of metabolic reactions, in addition to DNA and RNA biosynthesis, including protein biosynthesis, adenylosuccinate synthetase, ribonucleoside diphos- 


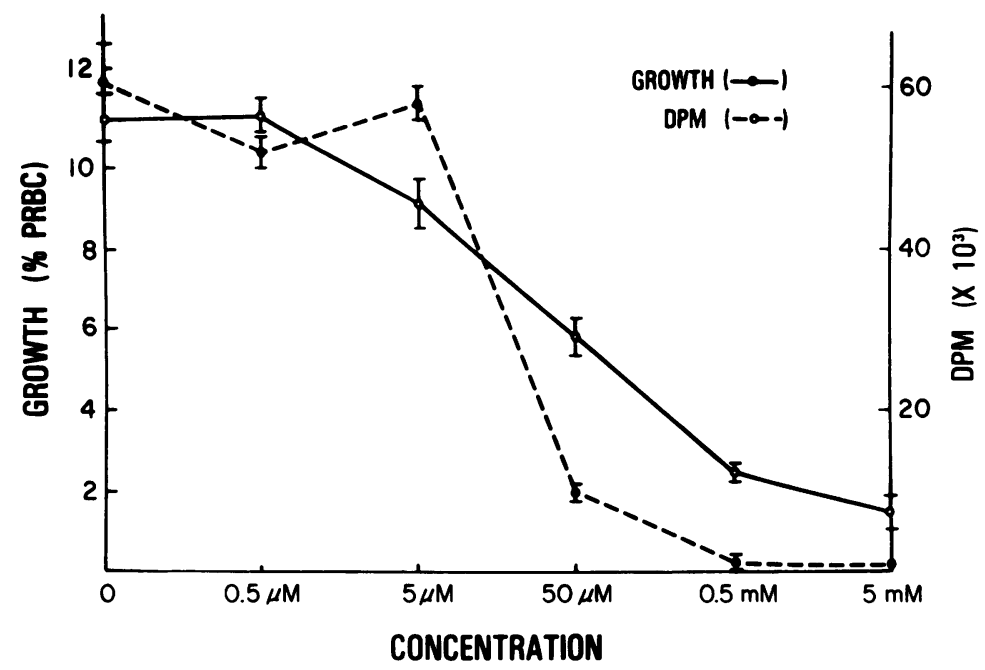

Figure 3 Microtiter assay for effect of bredinin on parasite growth (\% PRBC) and uptake of $\left[{ }^{3} \mathrm{H}\right]$ hypoxanthine $\left(\mathrm{dmp} \times 10^{3}\right)$. Semilogarithmic plot of bredinin molar concentration vs. parasite growth and uptake of $\left[\mathrm{G}-{ }^{3} \mathrm{H}\right]$ hypoxanthine. Disintegrations per minute indicate the uptake of labeled hypoxanthine by the parasite with increasing concentrations of bredinin. Parasite growth was determined on Giemsa-stained smears prepared from each microtiter well (expressed as number of PRBC per $100 \mathrm{RBC}$ ). The initial parasitemia was $0.5 \%$ PRBC $(n=3)$.

phate reductase, phosphoenolpyruvate carboxykinase, cytosine triphosphate synthetase, and polymerization of microtubules (16) -all of which are important for parasite growth and function. An intriguing possibility associated with impaired guanylate metabolism could involve the disruption of Plasmodia-specific synthesis of dihydrofolate from GTP. This assumes, however, that Plasmodia have a series of enzymatic reactions for synthesis of pteridine similar to that observed in bacteria and plants $(8,17)$. The loss of GTP would thus affect two parasite growth essential areas of metabolism-nucleic acids (DNA/RNA) and folates.

These studies demonstrate how basic biochemical information on intermediary metabolic pathways obtained through the use of human malaria culture techniques can lead directly to the identification of potential antimalarial agents. Through use of continuous RBC culture for $P$. falciparum (the major human malaria pathogen) it was possible to identify clearly the metabolic fate of the purine base, hypoxanthine. The simplicity of the culture system (RBC and malaria parasite) allowed detection of the purine pathways through which hypoxanthine was metabolized. Similarly, the control cultures permitted ready distinction of differences in metabolic pathways in unparasitized RBC as compared with malaria-infected RBC.

The identification of a metabolic pathway from hypoxanthine to guanosine nucleotides in PRBC, even though present to a limited extent in unparasitized RBC, made this pathway a logical selection as a che- motherapeutic target, based on the parasite's requirement for nucleic acid synthesis. The extensive literature on chemical agents examined for potential use in cancer chemotherapy made it possible to quickly identify compounds, like bredinin, known to affect guanylate metabolism in various experimental systems. A number of compounds other than bredinin have been shown to inhibit growth of tumor cells in tissue culture by interfering with synthesis of GMP from IMP: virazole, mycophenolic acid, psicofuranine, and decoyinine (18). We are presently examining several of these agents for antimalarial properties.

Although the antimalarial action of bredinin, demonstrated here in vitro, is as yet untested in vivo, the biochemical study of human malaria-infected RBC in vitro as described in this study, offers a rational approach to the discovery of potential metabolic targets for directed chemotherapy as well as the identification of new classes of candidate antimalarial drugs.

\section{ACKNOWLEDGMENTS}

We are indebted to Marvin Walker and Teri Bean for their excellent technical assistance, and to Joyce Powell and Vallapa Naewthai for typing the manuscript.

\section{REFERENCES}

1. Trager, W., and J. B. Jensen. 1976. Human malaria parasites in continuous culture. Science (Wash., DC). 193: 673-675.

2. Haynes, J. D., C. L. Diggs, F. A. Hines, and R. E. Des- 
jardins. 1976. Culture of human malaria parasites Plasmodium falciparum. Nature (Lond.). 263: 767-769.

3. Lowy, B. A., M. K. Williams, and I. M. London. 1962. Enzymatic deficiencies of purine nucleotide synthesis in human erythrocytes. J. Biol. Chem. 237: 1622-1627.

4. Bungener, W., and G. Nielson. 1967. Nucleic acid metabolism in experimental malaria (Plasmodium berghei and Plasmodium vinckei). Tropenmed. Parasitol. 18: 456-462.

5. Konigh, E. 1977. Salvage syntheses and their relationship to nucleic acid metabolism. Bull. W.H.O. 55: 249-252.

6. Sherman, I. W. 1979. Biochemistry of Plasmodium (malaria parasites). Microbiol. Rev. 43: 453-495.

7. Jung, H., R. Jackisch, A. Picard-Maureau, and R. Heishkeil. 1975. DNA, RNA, lipid synthesis, and the specific activity of glucose-6-phosphate dehydrogenase and glucose-6-phosphatase in various morphological stages of Plasmodium vinckei. Tropenmed. Parasitol. 26: 27-34.

8. Ferone, R. 1977. Folate metabolism in malaria. Bull. W.H.O. 55: 291-298.

9. Brewer, G. J. 1974. General red cell metabolism. In The Red Blood Cell. D. M. Surgenor, editor. Academic Press, Inc., New York. 2nd edition. 1: 387-433.

10. Webster, H. K., and J. M. Whaun. 1981. Purine metabolism during continuous erythrocyte culture of human malaria parasites ( $P$. falciparum). In The Red Cell (fifth Ann Arbor Conference). G. J. Brewer, editor. Alan R. Liss, Inc., New York. 557-570.

11. Sakaguchi, K., M. Tsuijino, M. Yoshigaiva, K. Mizuno, and K. Haynao. 1975. Action of bredinin on mammalian cells. Cancer Res. 35: 1643-1648.

12. Jensen, J. B., and W. Trager. 1978. Plasmodium falciparum in culture: establishment of additional strains. Am. J. Trop. Med. Hyg. 27: 743-746.

13. Canfield, C. J., E. G. Whiting, W. H. Hall, and B. MacDonald. 1971. Treatment of acute falciparum malaria from Vietnam with trimethoprim and sulfalene. Am. J. Trop. Med. Hyg. 20: 524-526.

14. Desjardins, R. E., C. J. Canfield, J. D. Haynes, and J. D. Chulay. 1979. Quantitative assessment of antimicrobial activity in vitro by a semiautomated microdilution technique. Antimicrob. Agents Chemother. 10: 710-718.

15. Webster, H. K., and J. M. Whaun. 1981. Application of simultaneous UV-radioactivity high performance liquid chromatography to the study of intermediary metabolism. I. Purines nucleotides, nucleosides, and bases. J. Chromatogr. 209: 283-292.

16. Lowe, J. K., L. Brox, and J. F. Henderson. 1977. Consequences of inhibition of guanine nucleotide synthesis by mycophenolic acid and virazole. Cancer Res. 37: 736743.

17. Brown, G. M. 1971. The biosynthesis of pteridines. Adv. Enzymol. 2: 35-77.

18. Suhadolinik, R. J. 1979. Naturally occurring nucleoside and nucleotide antibiotics. Prog. Nucleic Acid Res. Mol. Biol. 22: 193-291. 